\title{
Three-dimensional analysis of prebunched electron beams in an x-ray free-electron laser
}

\author{
H. P. Freund, D. C. Nguyen, and B. Carlsten \\ Los Alamos National Laboratory, Los Alamos, New Mexico 87545, USA
}

(Received 9 June 2011; published 12 March 2012)

\begin{abstract}
The effect of partial prebunching of an electron beam on the saturation length, efficiency, and longitudinal coherence is studied for a nominal x-ray free-electron laser design. Results indicate that partial prebunching of as little as $1 \%$ results in substantial (1) shortening of the saturation length, (2) increases in the efficiency, and (3) improvement in longitudinal coherence.
\end{abstract}

DOI: 10.1103/PhysRevSTAB.15.030704

PACS numbers: 41.60.Cr, 52.59.Rz

The recent success of the Linac Coherent Light Source (LCLS) at the Stanford Linear Accelerator Center [1] in producing coherent $x$ rays from a free-electron laser (FEL) has added impetus to the worldwide development of 4th generation light sources. The LCLS operates at a wavelength of $1.5 \AA$ and makes use of self-amplified spontaneous emission (SASE) in which shot noise on the beam is amplified in a single pass through the undulators. The main drawbacks to SASE are (1) a long undulator is required, (2) there is a relatively high level of shot to shot fluctuations, and (3) relatively poor longitudinal coherence. As a result, a number of alternative concepts are under consideration for x-ray FELs. These concepts can be grouped into three categories: (1) an oscillator design using Bragg crystal reflectors [2], (2) seeding with a coherent signal at a subharmonic and then employing a harmonic cascade [3], and (3) prebunching the electron beam prior to injection into the undulator [4]. In this paper, we focus on the concept of prebunching the electron beam.

Prebunching the electron beam is an established technique for increasing the efficiency and decreasing the interaction length in microwave tubes such as twystrons and inductive output tubes that employ gridded electron guns. More recently, a number of techniques have been proposed to prebunch the electron beam at shorter wavelengths such as x-ray wavelengths [4-6]. The concept relies on the fact that, if the beam is bunched on scale lengths comparable to or shorter than the desired wavelength, then the resonant wavelength is strongly excited without a drive signal. As a result, the output signal is temporally coherent and the interaction length is greatly reduced. Furthermore, with the strong interaction between the optical and prebunched electron beams, optical guiding can occur immediately after the coherent radiation is generated, thereby minimizing the interaction length and relaxing the beam emittance requirement. In this paper,

Published by the American Physical Society under the terms of the Creative Commons Attribution 3.0 License. Further distribution of this work must maintain attribution to the author(s) and the published article's title, journal citation, and DOI. we consider the efficacy of prebunched electron beams on the interaction in an x-ray FEL. However, due to incoherent spontaneous emission induced energy spread, it may be difficult to fully prebunch an electron beam at x-ray wavelengths; hence, we consider the effect of partial prebunching (consisting of a modulation of the electron bunch at the desired wavelength) on the interaction.

For simulation purposes, a prebunched beam model has been incorporated into the three-dimensional, polychromatic, time-dependent FEL simulation code MEDUSA [7]. MEDUSA [7-9] can model both planar and helical undulators and treats the electromagnetic field as a superposition of Gaussian modes. The field equations are integrated simultaneously with the three-dimensional Lorentz force equations. No wiggler-average orbit approximation is used, and MEDUSA can propagate the electron beam through a complex wiggler/transport line including multiple wiggler sections, quadrupole and dipole corrector magnets, FODO lattices, and magnetic chicanes. Since it is polychromatic and time dependent, MEDUSA can treat both sidebands and harmonic radiation, and macroparticles can be initialized in such a way as to simulate SASE [9]. MEDUSA has been successfully validated by comparison with several FEL oscillator [10], amplifier [11,12], and SASE experiments [13].

The prebunching model in MEDUSA [7] has been extended to describe partial prebunching in the phase space using a distribution of the form

$$
F(\psi)= \begin{cases}\frac{1-\Delta_{b}}{2 \pi}+\frac{2 \Delta_{b}}{\psi_{w}} \sin ^{2}\left(\frac{\pi \psi}{\psi_{w}}\right) ; & 0 \leq \psi \leq \psi_{w} \\ \frac{1-\Delta_{b}}{2 \pi} ; & \psi_{w}<\psi \leq 2 \pi\end{cases}
$$

which is illustrated in Fig. 1. Here $\psi_{w}(\leq 2 \pi)$ defines the width of the bunch, $\Delta_{b}$ denotes the bunching fraction which is defined as the ratio of the bunched charge [i.e., the area under the $\sin ^{2}$ function for which $0 \leq F(\psi)-$ $\left(1-\Delta_{b}\right) / 2 \pi \leq 2 \Delta_{b} / \psi_{w}$ ] to the total charge, and the integral of the distribution over the interval $[0,2 \pi]$ is unity. Observe that complete bunching is found in the limit as $\Delta_{b} \rightarrow 1$ while a uniform beam is recovered when $\Delta_{b} \rightarrow 0$. 


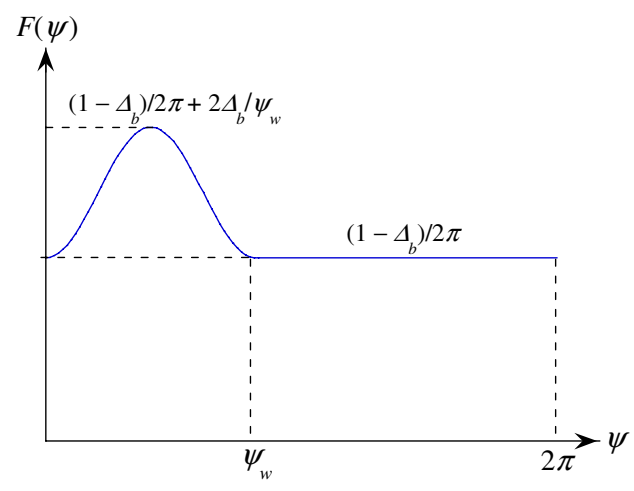

FIG. 1. Schematic of the partially prebunched phase space distribution.

The example under consideration is that of an $\mathrm{x}$-ray FEL operating at a wavelength of $0.248 \AA$. The electron beam has an energy of $20 \mathrm{GeV}$, a bunch charge of $100 \mathrm{pC}$, a bunch duration of $30 \mathrm{fsec}$, normalized emittances (in both the $x$ and $y$ directions) of $0.3 \mathrm{~mm} \mathrm{mrad}$, and an rms energy spread of $0.004 \%$. The undulator line is composed of 30 undulator segments each of which has a peak on-axis amplitude of $9.3 \mathrm{kG}$, a period of $2.4 \mathrm{~cm}$, and a length of 140 periods of which the first and last periods describe an up- and down-taper to match the electrons into and out of the undulator. The gaps between the undulators are $36 \mathrm{~cm}$ in length. A FODO lattice is composed of 29 quadrupoles located between the undulators (centered $21 \mathrm{~cm}$ from the end of the preceding undulator) each of which has a field gradient of $10.8 \mathrm{kG} / \mathrm{cm}$ and a length of $5 \mathrm{~cm}$. The Twiss parameters at the entrance to the undulator line have been chosen to give an optimal match through the lattice. This was found to occur for an initial rms beam size of $14.2 \mu \mathrm{m}$ in the $x$ direction and $13.2 \mu \mathrm{m}$ in the $y$ direction with $\alpha_{x}=$ 1.0 and $\alpha_{y}=-1.0$. Electron beam propagation through the undulator line for these Twiss parameters is illustrated in Fig. 2, where we plot the evolution in the beam envelopes in the $x$ (blue) and $y$ directions (red).

These are challenging, but achievable, beam parameters, since slice emittances of $0.3-0.4 \mathrm{mmmrad}$ have been achieved at the LCLS [1].

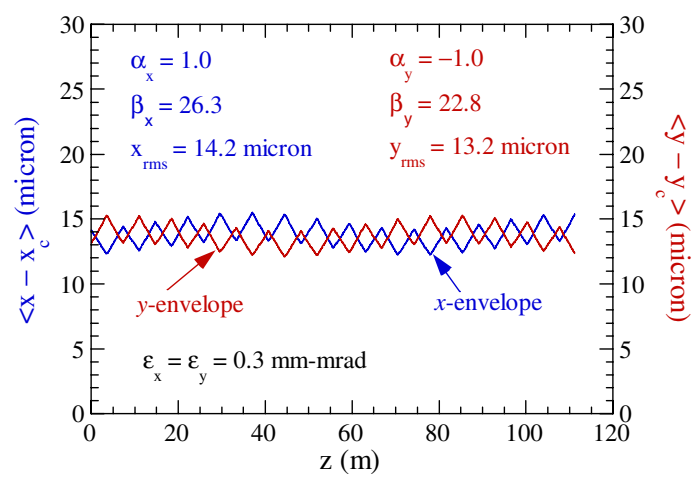

FIG. 2. Beam propagation through the undulator line.
An important issue in regard to the impact of partial prebunching is the degree of prebunching required to dominate over shot-noise driven SASE $[14,15]$. In order to address this issue, we consider the bunching parameter, which is defined as $b=\left|\left\langle e^{i \psi}\right\rangle\right|$. The bunching parameter (which is distinct from our use of the term "bunching fraction" to denote $\Delta_{b}$ ) can be calculated using the above initial phase distribution (1), and we find that

$$
b=\frac{4 \pi^{2} \Delta_{b}}{\psi_{w}\left(\psi_{w}+2 \pi\right)} \frac{\sin \Theta_{w}}{\Theta_{w}},
$$

where $\Theta_{w}=\left(\psi_{w}-2 \pi\right) / 2$. For the case under consideration here, we assume that $\psi_{w}=2 \pi$, so that $b=\Delta_{b} / 2$. In contrast, SASE is driven by shot-noise induced microbunching in phase, and the associated bunching parameter is proportional to the inverse root of the number of correlated electrons $\left(N_{e}\right)$, i.e., $b=1 / \sqrt{N_{e}}$. Since the number of correlated electrons is, typically, large, this means that even small levels of prebunching can have a significant effect. For example, the number of correlated electrons can be estimated as $[9,16]$

$$
N_{e}=4.3 \frac{L_{g} I_{b} \lambda}{\lambda_{w} e c}
$$

where $L_{g}$ is the e-folding length, $I_{b}$ is the peak current, $\lambda$ is the wavelength, $\lambda_{w}$ is the wiggler period, and $e$ and $c$ are the electronic charge and the speed of light in vacuo, respectively. For simplicity, and as we assume in the example herein, if we assume that the bunch width $\psi_{w}=2 \pi$, then the prebunching will dominate over SASE as long as

$$
\Delta_{b} \gg 0.96 \sqrt{\frac{\lambda_{w} e c}{L_{g} I_{b} \lambda}}
$$

For the example under consideration, the Pierce parameter $\rho \approx 3.5 \times 10^{-4}$ and the e-folding length is of the order of $4.3 \mathrm{~m}$; hence, $N_{e} \approx 1.4 \times 10^{6}$ and the shot-noise induced bunching parameter is $b \approx 8.5 \times 10^{-4}$. As a result, even bunching fractions of $0.5 \%$ or less can be expected to overwhelm the shot-noise induced SASE.

The principal point we address in this paper is the advantage partial prebunching confers in the saturation length, output power, and longitudinal coherence over the case of SASE. To this end, we have simulated the FEL for choices of $\Delta_{b}$ over the entire range from $0 \rightarrow 1$. The evolution of the x-ray pulse energy versus distance along the undulator line is shown in Fig. 3. Here we consider pure SASE and partial prebunching of $1 \%, 10 \%, 40 \%$, and $100 \%$, respectively. It is evident in the figure that even partial prebunching results in substantially shorter saturation distances and higher output energies.

Pure SASE saturates after a distance of about $95 \mathrm{~m}$ with pulse energy of $0.48 \mathrm{~mJ}$. For the pulse length of $30 \mathrm{fsec}$, this is equivalent to a power of about $16 \mathrm{GW}$. The e-folding 


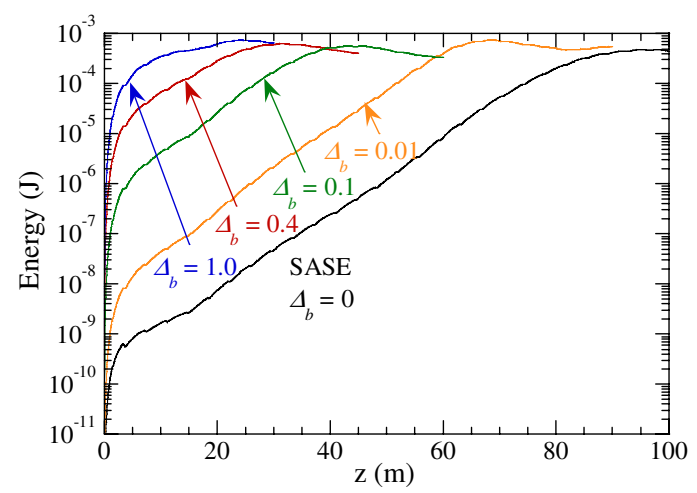

FIG. 3. Evolution of the x-ray pulse energy for pure SASE and for several choices of the bunching fraction.

length is about $5.7 \mathrm{~m}$. Analytic estimates of the performance [17] predict an e-folding length of $4.4 \mathrm{~m}$ and a saturated power of $22 \mathrm{GW}$, which is in reasonable agreement with the simulation. In contrast, complete prebunching $\left(\Delta_{b}=1.0\right)$ saturates after $24 \mathrm{~m}$ with a pulse energy of $0.69 \mathrm{~mJ}$. Intermediate levels of partial prebunching require longer saturation distances, but even $1 \%$ prebunching results in a substantially shorter saturation distance than pure SASE.

A summary of the variation in the x-ray pulse energy (circles) and saturation distance (diamonds) versus bunching fraction is shown in Fig. 4. It is clear from the figure that the saturation distance initially decreases sharply with increasing bunching fraction but rolls over to a more gradual decline once the bunching fraction increases beyond about $30 \%$. Nevertheless, the saturation distance is about $69 \mathrm{~m}$ for a bunching fraction of $1 \%$, which is still substantially shorter than that for pure SASE. It is also apparent from the figure that the X-ray pulse energy even for partial prebunching is also substantially increased over pure SASE, and that a peak of about $0.75 \mathrm{~mJ}$ is found for a bunching fraction of about $1 \%$ and varies weakly with bunching fraction as $\Delta_{b}$ increases to unity.

An important characteristic of the output pulse is the longitudinal coherence. To address this issue, we plot the

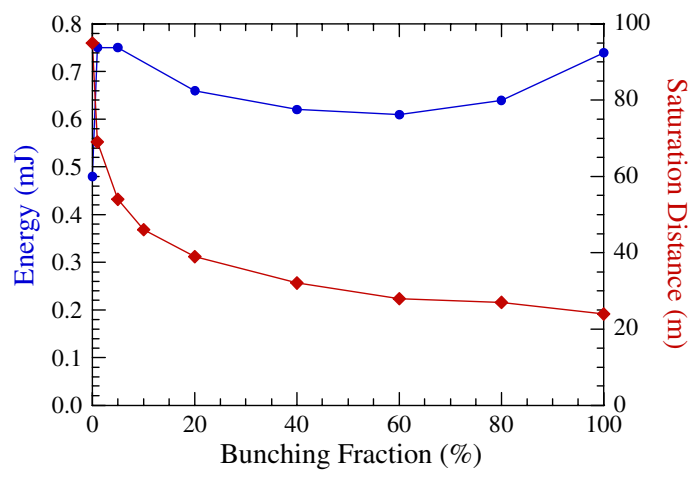

FIG. 4. Variation in the x-ray pulse energy at saturation and saturation distance versus bunching fraction.

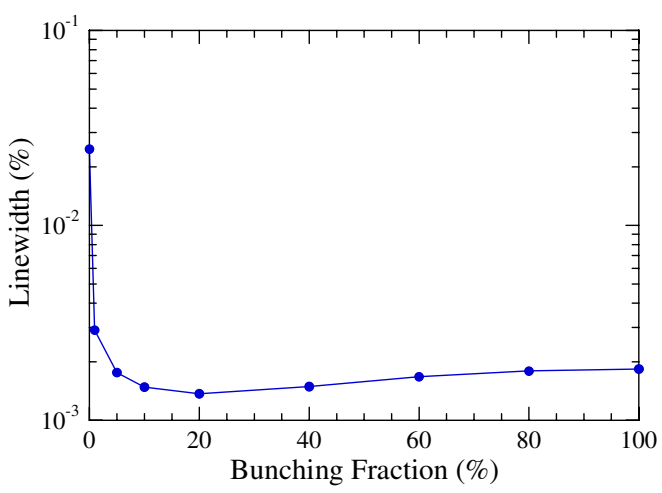

FIG. 5. Variation in the linewidth at saturation versus the bunching fraction.

rms linewidth (defined as $\Delta \lambda_{\text {rms }} / \lambda_{\text {avg, where }} \lambda_{\text {avg }}=$ $0.248 \AA$ ) of the output radiation at saturation versus the bunching fraction in Fig. 5. It is clear from the figure that the linewidth drops rapidly as the bunching fraction increases from zero. The linewidth for SASE found in simulation is $\Delta \lambda_{\mathrm{rms}} / \lambda_{\mathrm{avg}}=2.46 \times 10^{-4}$ at saturation, which is close to the theoretical value of $2.87 \times 10^{-4}$ [18]. The linewidth decreases by almost an order of magnitude as $\Delta_{b}$ increases to $1 \%$ where $2.90 \times 10^{-5}$ at saturation. This is because even $1 \%$ prebunching overwhelms the shot noise and results in a coherent interaction. The linewidth decreases further as $\Delta_{b}$ increases to $10 \%$ at which point $\Delta \lambda_{\text {rms }} / \lambda_{\text {avg }}=1.48 \times 10^{-5}$, after which it is relatively insensitive to increases in $\Delta_{b}$ where $\Delta \lambda_{\text {rms }} / \lambda_{\text {avg }}$ varies within the range of about $1.49 \times 10^{-5}-1.84 \times 10^{-5}$. This narrowing of the linewidth with partial prebunching is shown dramatically in Figs. 6 and 7 where we plot the spectra at saturation for pure SASE and for a bunching fraction of $1 \%$, respectively.

The sensitivity of the interaction to the energy spread both for SASE and for partially prebunched beams is illustrated in Fig. 8, where we plot the normalized efficiency (defined as the ratio of the efficiency at the specified energy spread to the efficiency in the absence of an energy spread). The case of a vanishing bunching fraction, as

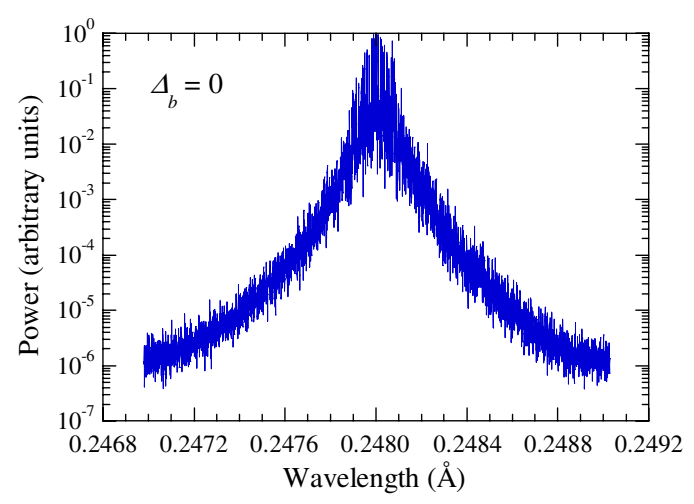

FIG. 6. Spectrum of pure SASE at saturation. 


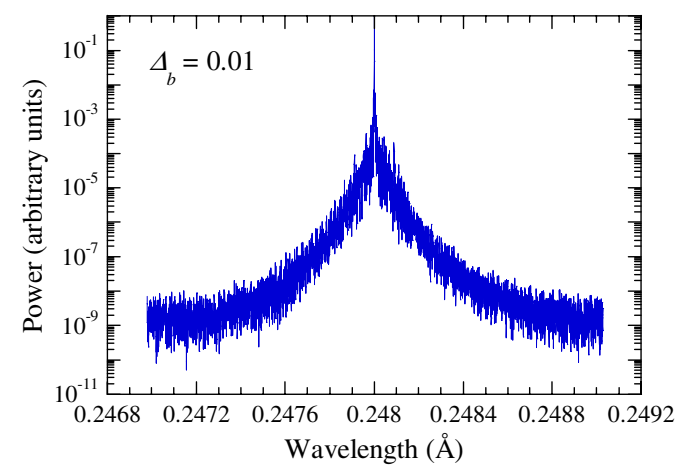

FIG. 7. Spectrum at saturation for a bunching fraction of $1 \%$.

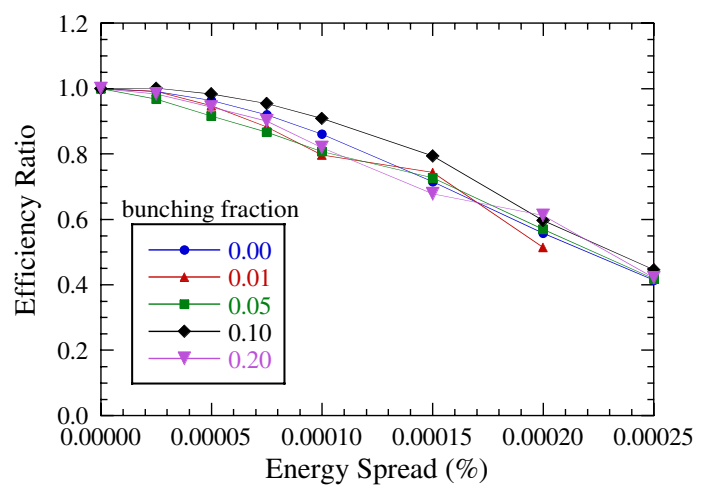

FIG. 8. Effect of the energy spread on the efficiency.

before, corresponds to SASE. The points on the curve for the case of SASE were obtained from analytic theory [17] while those for bunching fractions in the range of 1\%-20\% were found from simulation. It is evident from the figure that the sensitivity of the interaction to increasing energy spread is comparable for SASE as well as for prebunched beams.

In summary, we have examined the effect of prebunching on the output pulse energy, saturation length, and longitudinal coherence in an X-ray FEL. While the specific parameters considered herein are challenging, we believe that they are achievable; however, the basic effects of even partial prebunching are relevant to FELs operating at any wavelength with a wide variety of electron beam parameters. The results indicate that substantial (1) increases in output pulse energy, (2) shortening in the saturation length, and (3) improvements in longitudinal coherence are possible with even modest levels of prebunching (i.e., $1 \%$ or less). Indeed, if the bunching ratio for the partial prebunching exceeds that for SASE, then the SASE is overwhelmed and the effect of increasing the bunching fraction is relatively mild. In view of the fluctuations in the output of SASE FELs in terms of both power and spectral properties, the ability to partially prebunch an electron beam prior to injection into the undulator line may represent the next generation in coherence light sources.

\section{ACKNOWLEDGMENTS}

The work was supported in part by the Office of Naval Research and by the U.S. Department of Energy through the LANL/LDRD program. Computer time was provided by Jefferson Laboratory, operated by Jefferson Science Associates, LLC under U.S. DOE Contract No. DEAC05-06OR23177.

[1] P. Emma et al. (LCLS team), in Proceedings of the 23rd Particle Accelerator Conference, Vancouver, Canada, 2009 (IEEE, Piscataway, NJ, 2009), H3PBI01.

[2] R. R. Lindberg et al., Phys. Rev. ST Accel. Beams 14, 010701 (2011).

[3] L.-H. Yu et al., Science 289, 932 (2000).

[4] S. J. Russell et al., in Proceedings of the 23rd Particle Accelerator Conference, Vancouver, Canada, 2009 (Ref. [1]), TH5PFP036.

[5] G. Stupakov, Phys. Rev. Lett. 102, 074801 (2009).

[6] B. Carlsten et al. (unpublished).

[7] H.P. Freund et al., Nucl. Instrum. Methods Phys. Res., Sect. A 507, 400 (2003).

[8] H. P. Freund, Phys. Rev. ST Accel. Beams 8, 110701 (2005).

[9] H. P. Freund, L. Giannessi, and W. H. Miner, Jr., J. Appl. Phys. 104, 123114 (2008).

[10] P. J. M. van der Slot et al., Phys. Rev. Lett. 102, 244802 (2009).

[11] X. J. Wang et al., Appl. Phys. Lett. 91, 181115 (2007).

[12] X. J. Wang et al., Phys. Rev. Lett. 103, 154801 (2009).

[13] L. Giannessi et al., Phys. Rev. ST Accel. Beams 14, 060712 (2011).

[14] L. H. Yu and S. Krinsky, Nucl. Instrum. Methods Phys. Res., Sect. A 285, 119 (1989).

[15] K.-J. Kim, Nucl. Instrum. Methods Phys. Res., Sect. A 393, 167 (1997).

[16] L. Giannessi, in Proceedings of FEL2004 Conference (Comitato Conferenze Elettra, Trieste, Italy, 2004), p. 37.

[17] M. Xie, in Proceedings of the Particle Accelerator Conference, Dallas, TX, 1995 (IEEE, New York, 1995), Catalog No. 95CH35843, p. 183.

[18] Z. Huang and K.-J. Kim, Phys. Rev. ST Accel. Beams 10, 034801 (2007). 